\title{
A Photoredox Coupling Reaction of Benzylboronic Esters and Carbonyl Compounds in Batch and Flow
}

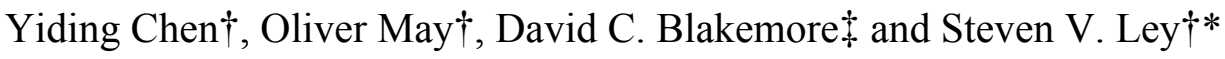 \\ $\uparrow$ Department of Chemistry, University of Cambridge, Lensfield Road, Cambridge CB2 1EW, U.K. \\ \$ Medicine Design, Pfizer Inc., Eastern Point Road, Groton, Connecticut 06340, United States
}

Supporting Information Placeholder

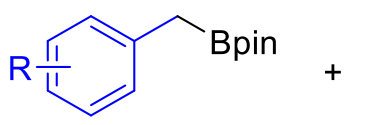<smiles>[R]C(=[X])c1ccccc1</smiles>
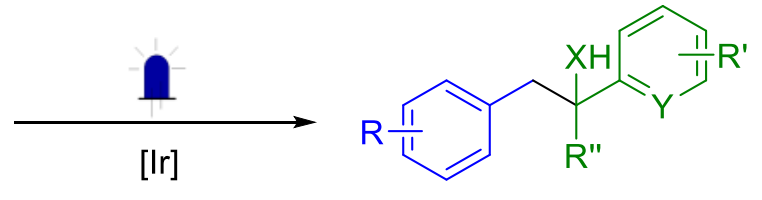

$\mathrm{X}=\mathrm{N}$ or $\mathrm{O}$
$\mathrm{Y}=\mathrm{CH}$ or $\mathrm{N}$

\begin{abstract}
Mild cross-coupling reaction between benzylboronic esters with carbonyl compounds and some imines was achieved under visible-light induced iridium-catalyzed photoredox conditions. Functional group tolerance was demonstrated by 51 examples, including 13 heterocyclic compounds. Gram-scale reaction was realized through the use of computer-controlled continuous flow photoreactors.
\end{abstract}

Carbonyl compounds serve as fundamentally important electrophilic building blocks during carbon-carbon bond forming reactions commonly using transition metal catalysis ${ }^{1}$ or organometallic coupling processes. ${ }^{2}$ While wide functional group tolerance can be achieved in these reactions, ${ }^{3}$ there is interest in moving away from using strongly basic conditions or highly reactive substrates. ${ }^{4}$ In this content, the current trend in the use of visible light photoredox methods has led to impressive discoveries, especially to bring about carbon-carbon bond formation with single electron transfer processes. ${ }^{5}$

Previously we have noted the addition of Lewis bases to activate photoredox mediated coupling reactions between normally unreactive benzylic boronic esters and olefinic acceptors. ${ }^{6}$ Others have also reported useful processes whereby selective reductive addition to carbonyl compounds can be achieved. ${ }^{7}$ More specifically, Rueping described the dimerization of aldehydes and ketones via intermediate photoredox generated ketyl radicals. ${ }^{8}$ Several other reports ${ }^{9}$ concerning reactions with alkenes, ${ }^{10}$ intramolecular cyclisation ${ }^{11}$ or photocatalytic Barbier type reactions ${ }^{12}$ via putative radical-radical coupling pathways are known as well. In view of the opportunities that arise through these photoredox coupling processes, we commenced the study below employing benzylic boron esters as precursors during this potential direct coupling with carbonyl compounds and imines, thus avoiding strongly nucleophilic organometallic reagents.

From a test reaction based on the conditions we had explored previously, ${ }^{6}$ we were pleased to observe an optimal yield of $84 \%$ for the specific cross coupling event of the $p$-methoxybenzylboronic acid pinacol ester 1a with $p$-chlorobenzaldehyde $\mathbf{2 a}$. This was achieved by employing iridium photoredox catalyst $\mathbf{i}$ with 3-quinuclidinol in a 0.05 M 1:1 methanol and acetone solvent system under a blue LED light for 8 hours to give the addition product 3a (Table 1, entry 1). For full details of the optimization and screening see Supporting Information. Although these conditions bear a similarity to recent procedures, ${ }^{6}$ the reaction progressed rather differently over the optimization process. Commonly used photocatalysts mesityl acridinium salt ii and the ruthenium catalyst iii failed to deliver coupled products (entry 2 and 3). On the other hand, using 4-dimethylaminopyridine (DMAP) as additive a moderate $44 \%$ of product 3a was realized (entry 4), while addition of the Lewis acid $\mathrm{Sc}(\mathrm{OTf})_{3}$ together with $20 \mathrm{~mol} \%$ equivalent of the quinuclidine base did not significantly enhance the overall reactivity (entry 5). Solvent changes were usually detrimental; for example, acetonitrile leads to a yield of only $19 \%$ of the coupled product (entry 6). However, it is worth noting that the concentration of the reaction mixture does have a noticeable impact, in that a 0.1 $M$ reaction mixture caused a drop in yield to $54 \%$ (entry 7 ). More difficult to understand in this specific coupling example was the $43 \%$ yield of product 3a when no base was added to the mixture (entry 8), about which is discussed later. Without either photocatalyst or a light source the reaction was completely shut down (entry 9 and 10). Switching to green light led to a decreased yield of $16 \%$ (entry 11$)$. 
Table 1. Reaction optimisation

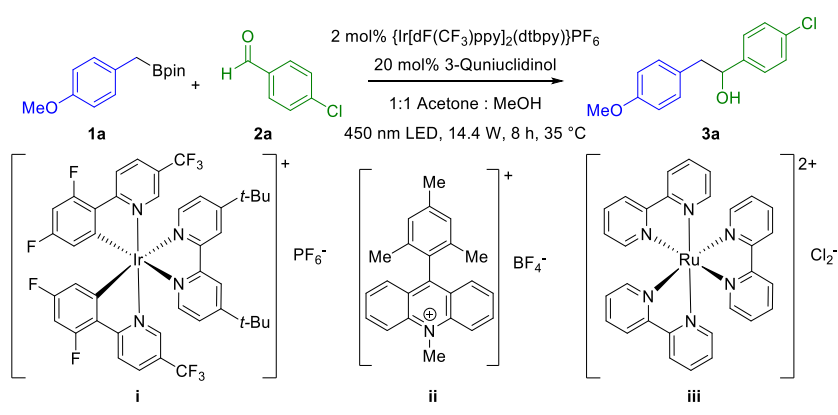

$\begin{array}{lll}\text { Entry }^{[\mathrm{a}]} & \text { Variation from above conditions } & \text { Yield }^{[\mathrm{b}]} \\ 1 & \text { No variation } & \begin{array}{l}86 \% \\ (84 \%)^{[\mathrm{c}]}\end{array} \\ 2 & 2 \mathrm{~mol} \% \text { Mes-Acr-BF } 4 \text { (ii) instead of Ir } & 24 \% \\ 3 & 2 \mathrm{~mol} \%\left[\mathrm{Ru}(\mathrm{bpy})_{3}\right] \mathrm{Cl}_{2} \text { (iii) instead of } \mathrm{Ir} & 0 \% \\ 4 & 50 \mathrm{~mol} \% \text { DMAP as base } & 44 \% \\ 5 & \text { Additional } 20 \text { mol\% Sc(OTf) } & 70 \% \\ 6 & \text { Acetonitrile }(0.1 \mathrm{M}) \text { as solvent } & 19 \% \\ 7 & \text { 0.1 M concentration instead of } 0.05 \mathrm{M} & 54 \% \\ 8 & \text { Without base } & 43 \% \\ 9 & \text { Without photocatalyst } & 0 \% \\ 10 & \text { Without light } & 0 \% \\ 11 & \text { Green LEDs (540 nm, 14 W) } & 16 \%\end{array}$

[a] Reaction conditions: $0.1 \mathrm{mmol}$ boronic acid pinacol ester, $0.15 \mathrm{mmol}$ aldehyde, $1 \mathrm{~mL}$ acetone and $1 \mathrm{~mL}$ methanol. ${ }^{[\mathrm{b}]} \mathrm{NMR}$ yield using 1,3,5-trimethoxybenzene as an internal standard. ${ }^{[\mathrm{c}]}$ Isolated yield

With the conditions from entry 1 in hand, we directed our attention to examine a series of benzyl boronic esters $\mathbf{1}$ and coupling partners 2 (Figure 1). Generally, electron donating group (EDG) substituents on the boronic ester component worked well, as exemplified by high yields from the optimized 4-methoxyphenyl substrate (1a) as well as $p$-methylbenzyl (1b), omethylbenzyl (1c) and 1-nathphylmethyl (1d) boronic esters. The parent benzyl boronic ester (1e) also provided moderate yield of coupled product (3e), although electron withdrawing groups (EWG) such as the $p$-trifluoromethoxybenzyl derivative (1f), $p$-chlorobenzyl (1g), $m$-fluorobenzyl (1h), $p$-methylbenzoate (1i) all showed lower efficiencies. Likewise, for the phenylsulfanylmethyl boronic ester (1j), only $16 \%$ of the corresponding coupled product (3j) was obtained. Similarly, with other long-chain aliphatic boronic esters and aryl boronic esters, no useful products were observed.

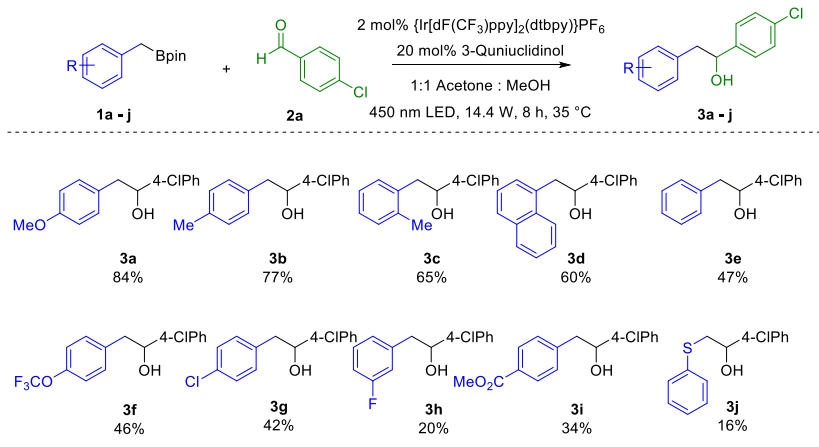

Figure 1. Scope of boronic acid pinacol esters.

To further investigate the scope with respect to aldehydes 2 (Figure 2), we examined a wide range of aromatic aldehydes with various ring substituents $(\mathbf{2} \mathbf{k}-\mathbf{z})$. Among these a number contain challenging features, such as bromo- (2l), boc-protected amino- (2m), photosensitive nitrile (2n), boron pinacol ester (2o), hydroxyl (2p) and pentafluoro (2y) groups. Also interestingly, given a choice between a ketone and aldehyde as coupling partners, we saw complete aldehyde specificity (3s). In three examples, addition to imines occurs readily to afford coupled products $(3 \mathbf{v}-\mathbf{x})$. Particularly worth noting is that the method is compatible with many heterocyclic substrates. Excellent yields were obtained from pyridyl substrates (3aa-3ad). The nitrogen-containing heterocycles pyrrole, pyrimidine and quinoline (3ae- 3ag), as well as sulfur-containing heterocyclic compounds such as thiazole (3ah) and thiophene (3ai and 3aj), all reacted satisfactorily. For completeness, listed in red are a few aldehyde coupling partners that failed to yield useful products.

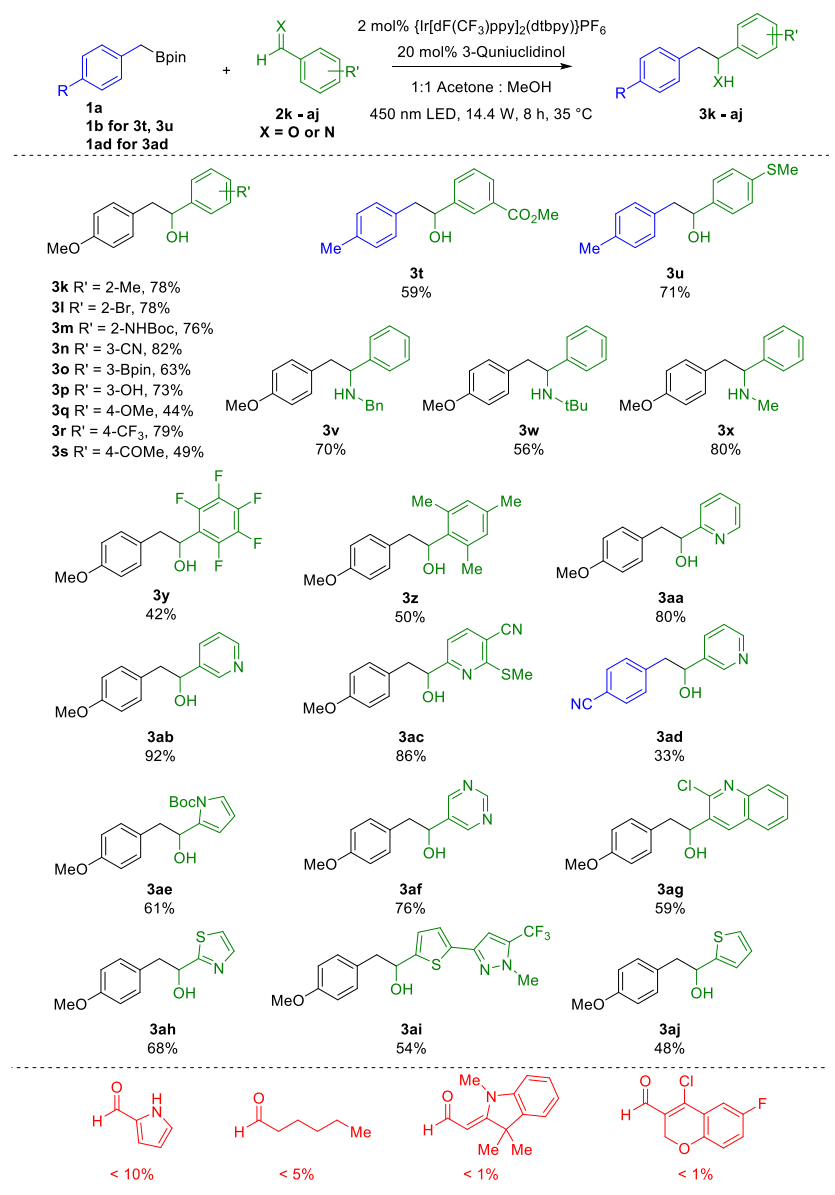

Figure 2. Scope of aryl and heteroaryl aldehydes, and imines. 
We further expanded the scope of the reactions to include ketone derivatives (Figure 3). Acetophenone derivatives $(\mathbf{4 a}-\mathbf{4 g})$ were tolerated, while better yields were observed with benzophenone substrates $(\mathbf{5 h}-\mathbf{5 j})$. Photochemically-active reagents such as xanthenone (5k), thioxanthenone (5l) and fluorenone (5m) were particularly reactive, which supports our later mechanistic proposals. Acetohexamide (4n) ${ }^{13}$ successfully delivered the coupled product in $59 \%$ yield (5n). In this example, a reversed ratio of 1.5:1 boronic ester and ketone was employed to aid with the isolation of the benzylated product from acetohexamide starting material. In cases where there is significant enolization in the coupling partner (shown in red), very poor product yields are realized.

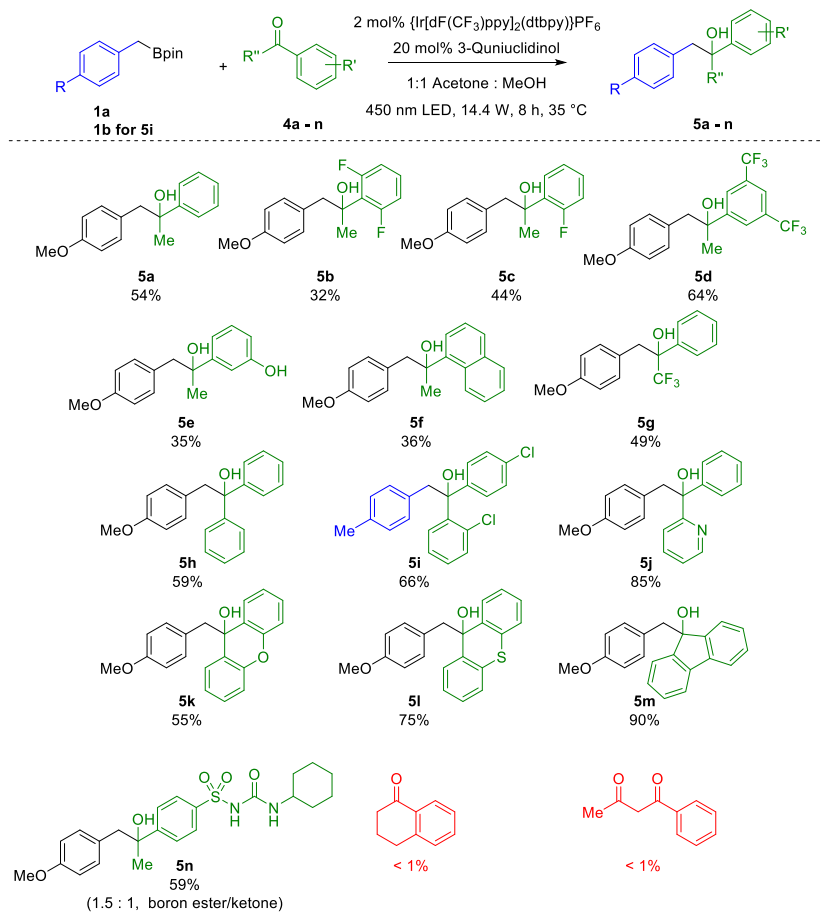

Figure 3. Scope of ketones.

The use of continuous methods (Scheme 1$)^{14}$ has been shown to offer immediate improvements over equivalent batch photoredox procedures ${ }^{15}$ when scaling reactions, particularly when new reactor designs lead to improved photon flux densities ${ }^{16}$ and material throughput. ${ }^{17}$
Reaction mixtures were prepared in a normal fashion and pumped through a photoreactor (Vapourtec UV-150) fitted with a blue LED module $(17 \mathrm{~W}$ at $\lambda=420 \mathrm{~nm})$ and a $10 \mathrm{~mL}$ coil (FEP, 1/16"). With a residence time of $100 \mathrm{~min}, 100 \%$ conversion was obtained giving a $70 \%$ 3a isolated yield (Scheme 1a). Encouraged by this early success, a larger scale experiment was carried out on the recently commercialized Uniqsis Photosyn reactor, which was equipped with a more powerful $(420 \mathrm{~W}$ at $\lambda$ $=450 \mathrm{~nm}$ ) lamp and a bigger $20 \mathrm{~mL}$ (PFA, 1/16") flow coil, which could further reduce the residence time to $60 \mathrm{~min}$. The reaction was controlled with LabVIEW program and was monitored with Uniqsis Flow-UV. A 10-hour run was carried out to produce $1.69 \mathrm{~g}$ of product (3ak). (See Supporting Information for detailed reaction set-up, no attempt at this stage was made to scale up the reaction any further).

Scheme 1. Continuous flow synthesis. a) With Vaportec UV-150. b) With Uniqsis Photosyn controlled by LabVIEW.
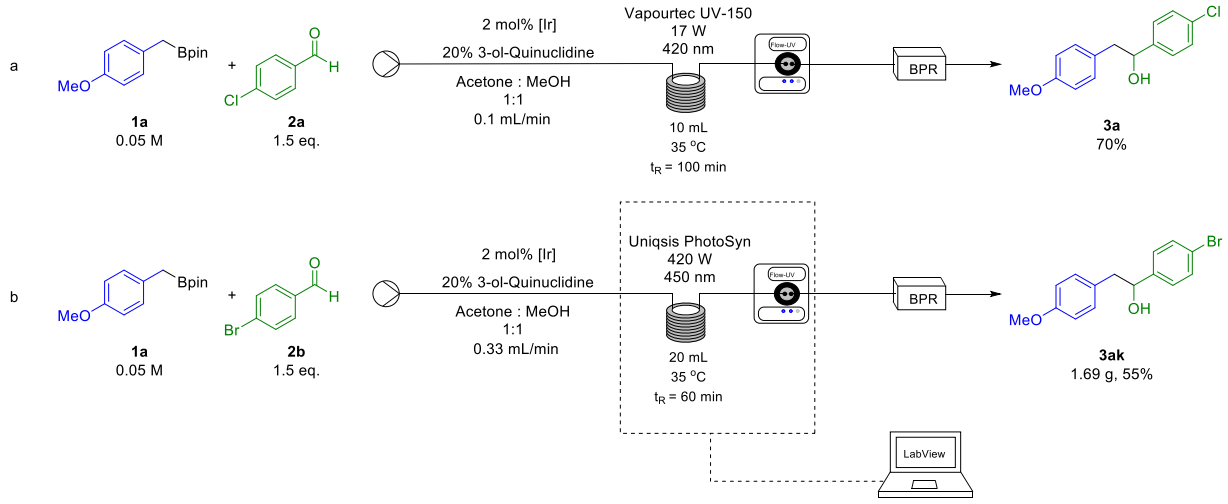
As mentioned above, this reaction was performed under similar catalytic conditions to our previous work. ${ }^{6}$ However, two main differences in reactivity were noticed. Firstly, while aromatic boronic acid pinacol esters and aliphatic boronic acids were viable precursors in our earlier study, ${ }^{4}$ only benzylic Bpin esters were successful in the current work. Additionally, previous studies clearly demonstrated that other Lewis bases (e.g. DMAP) could be as efficient as quinuclidine, whereas in this new work DMAP did not promote the reaction to any equivalent level (Table 1, entry 4). These distinctive differences, though not direct evidence, suggested a mechanism that differed from a Lewis base-catalyzed photoredox activation of the boron species. $^{6}$

In other control experiments (Table 1 , entry 9 and 10 , see more in Supporting Information) suggested the iridium complex $\mathbf{i}$ was the only functional photoredox catalyst, while no conclusion can be drawn about the absolute necessity of quinuclidine-ol additive (Table 1, entry 8 ). This observation contrasts with previous mechanistic proposals ${ }^{18}$ since the reduction potential of most carbonyl compounds presented are beyond the range of this particular photocatalyst. ${ }^{19}$ For example, the reductive potential of benzophenone is $\left(\mathrm{E}_{1 / 2}{ }^{\mathrm{Red}}=-1.87 \mathrm{~V}\right.$ vs SCE$)$ whereas the reductive potential of the catalyst $\mathbf{i}$ is $\left(E_{1 / 2}{ }^{\operatorname{Red}}=-1.37 \mathrm{~V}\right.$ vs $\mathrm{SCE}){ }^{20}$ Hence a further control experiment was performed (Scheme 2a). Using 4-methylbenzyl boronic pinacol ester $\mathbf{1 b}$ as precursor, only $6 \%$ desired product $3 \mathrm{~b}$ was detected in the absence of a base additive. This contrasts with a $77 \%$ yield when a $20 \mathrm{~mol} \%$ 3-quinuclidin-ol was added (Figure 1, 3b), indicating that the quinuclidine base plays a crucial role. These results might suggest that the methoxyboronic ester could initiate reaction activation, where similar observation was reported by König et. al. ${ }^{21}$ Competition reactions between chlorobenzaldehyde and ethyl acrylate as coupling partners were also briefly investigated (Scheme $2 \mathrm{~b}$ ). In the presence of quinuclidine base, $61 \%$ of the coupled boronic ester 7 was realized and only $8 \% \mathbf{3 a}$ was isolated. Lastly, the product yield dropped dramatically with the addition of radical scavenger TEMPO (Scheme 2c), further proving the existence of a radical pathway.
Scheme 2. Additional control experiment. a) Coupling of pmethylbenzylboronic ester with p-chlorobenzaldehyde in the absence of quinuclidine. b) Competition reaction between benzaldehyde and ethyl acrylate with benzylboronic ester. c) Radical trap experiment.

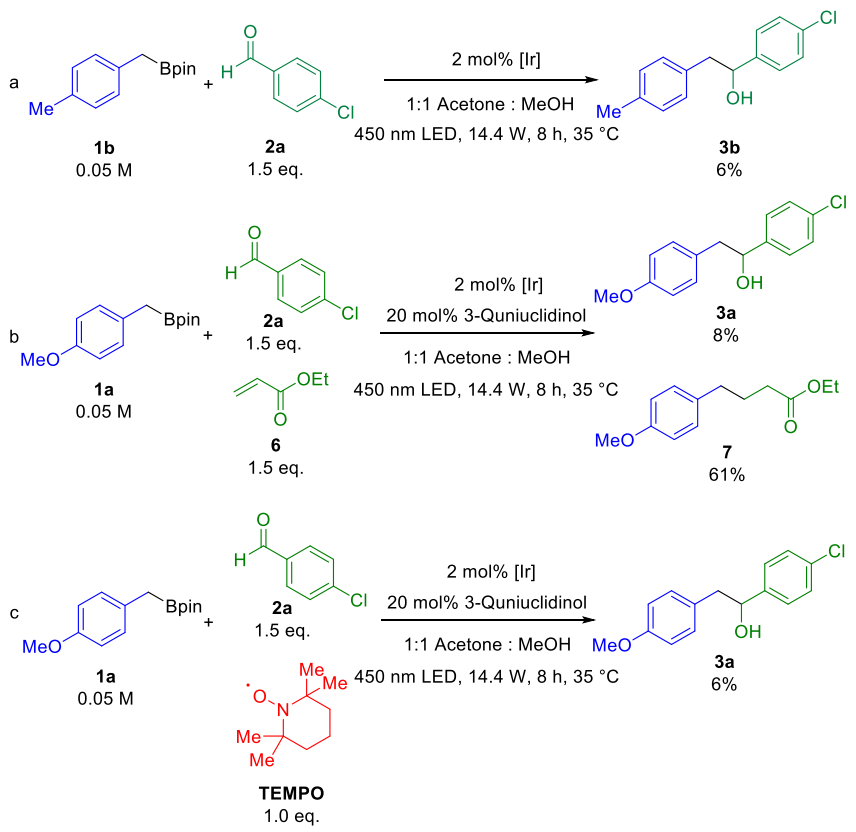

The reaction analysis may suggest a ketyl radical pathway could also be operating. In most cases a byproduct formed in the reaction which was thought to be a diol from aldehyde/ketone dimerization, as expected from a ketyl radical mechanism. To the best of our knowledge, although radical trapping with carbonyl compounds are known, ${ }^{7 \mathrm{e}, 22}$ the generation of thermodynamically unfavorable alkoxy radical often lead to a reversable process ${ }^{23}$ or homolytic $\mathrm{C}-\mathrm{C} \beta$-scission. ${ }^{24}$

We therefore propose the mechanism shown in Figure 4. Initially, the Ir(III) catalyst $\mathbf{i}$, when exposed to blue light, is excited to a long-lifetime excited state Ir(III)*. ${ }^{20}$ Quinuclidine base $\left(\mathrm{E}_{1 / 2}{ }^{\mathrm{Ox}}=+1.00 \mathrm{~V} \text { vs SCE}\right)^{25}$ then undergoes single electron oxidation by the photo-excited $\operatorname{Ir}(\mathrm{III}) *$ catalyst $\left(\mathrm{E}_{1 / 2}{ }^{\mathrm{Ox}}=+1.21 \mathrm{~V}\right.$ vs $\mathrm{SCE}),{ }^{20}$ leading to the initiation of radical cation $\mathbf{A}$ and the reduced Ir(II) complex. ${ }^{26}$ The aldehyde/ketone activated by boronic ester ${ }^{21}$ is then reduced by $\mathrm{Ir}(\mathrm{II})$ complex $\left(\mathrm{E}_{1 / 2}{ }^{\mathrm{Red}}=-1.37 \mathrm{~V}\right.$ vs SCE) via single electron reduction thus completing the photoredox cycle. The resulting ketyl radical anion $\mathbf{B}$ subsequently attacks the benzylboronic acid pinacol ester to assemble the borate radical anion $\mathbf{C}$. Alternatively, the aldehyde/ketone could be activated by boronic ester through a boron-oxygen interaction thus the single electron reduction creates radical anion $\mathbf{C}$ directly. $\mathbf{C}$ is then oxidized by radical cation $\mathbf{A}$, which terminates the radical sequence and to give borate $\mathbf{D}$ and the quinuclidine catalyst. Finally, D is hydrolyzed to the targeted alcohol product. 


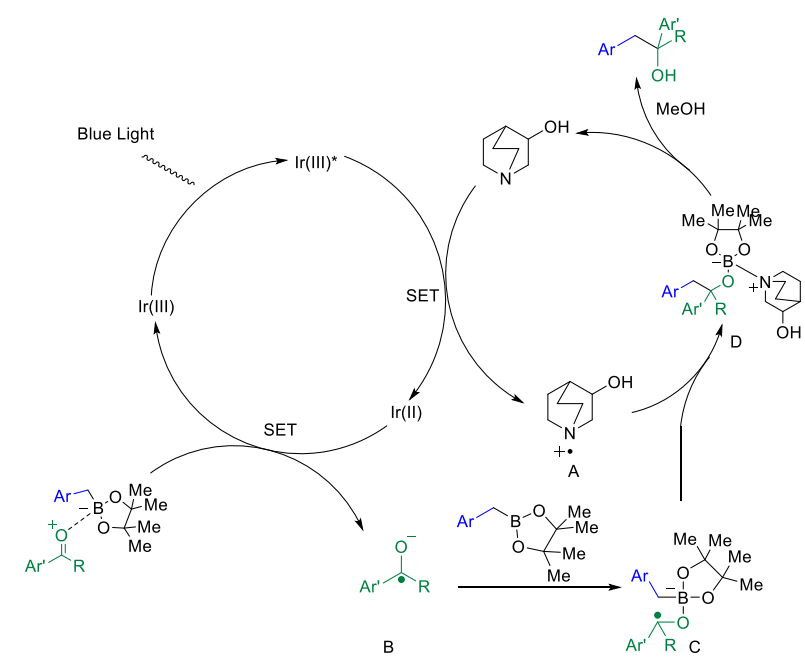

Figure 4. Proposed mechanism

In summary, we describe above a reductive cross-coupling reaction of benzylic organoboron reagents with carbonyl compounds via photoredox catalysis, displaying wide functional group tolerance. Preliminary results also show the reactions could be scaled using more powerful light sources coupled to computer-controlled flow chemistry devices.

\section{ASSOCIATED CONTENT}

\section{Supporting Information}

The Supporting Information is available free of charge on the ACS Publications website.

Details of experimental procedure, compound characterization data and NMR spectra (PDF)

\section{AUTHOR INFORMATION}

\section{Corresponding Author}

*Email:sv11000@cam.ac.uk

\section{ORCID}

Yiding Chen: 0000-0001-6932-9005

Steven V. Ley: 0000-0002-7816-0042

\section{Notes}

The authors declare no competing financial interest other than D.C.B. is an employee and stockholder of Pfizer Inc. Additional data related to this publication is available at the University of Cambridge Institutional Data Repository doi.org/10.17863/CAM.40213

\section{ACKNOWLEDGMENT}

We thank Uniqsis Ltd and Mark Ladlow for the generous loan of a Photosyn reactor. Y. C. thanks Pfizer for funding the postdoctoral fellowship. The authors also gratefully acknowledge financial support from H2020-FETOPEN-2016-2017 programme of European commission (SVL; grant agreement number: 737266-ONE FLOW).

\section{REFERENCES}

1. (a) Kuriyama, M.; Shimazawa, R.; Shirai, R. J. Org. Chem. 2008, 73, 1597-1600; (b) Gois, P. M. P.; Trindade, A. F.; Veiros, L. F.; André,
V.; Duarte, M. T.; Afonso, C. A. M.; Caddick, S.; Cloke, F. G. N. Angew. Chem. Int. Ed. 2007, 46, 5750-5753.

2. Hollerbach, M. R.; Barker, T. J. Organometallics 2018, 37, 14251427

3. Sakai, M.; Ueda, M.; Miyaura, N. Angew. Chem. Int. Ed. 1998, 37, 3279-3281.

4. Yamamoto, Y.; Asao, N. Chem. Rev. 1993, 93, 2207-2293.

5. (a) Campos, K. R.; Coleman, P. J.; Alvarez, J. C.; Dreher, S. D.; Garbaccio, R. M.; Terrett, N. K.; Tillyer, R. D.; Truppo, M. D.; Parmee, E. R. Science 2019, 363, eaat0805; (b) Banerjee, A.; Lei, Z.; Ngai, M.Y. Synthesis 2019, 51, 303-333; (c) Shu, C.; Noble, A.; Aggarwal, V. K. Angew. Chem. Int. Ed. 2019, 58, 3870-3874; (d) Shaw, M. H.; Shurtleff, V. W.; Terrett, J. A.; Cuthbertson, J. D.; MacMillan, D. W. C. Science 2016, 352, 1304-1308; (e) Le, C.; Liang, Y.; Evans, R. W.; Li, X.; MacMillan, D. W. C. Nature 2017, 547, 79.

6. Lima, F.; Sharma, U. K.; Grunenberg, L.; Saha, D.; Johannsen, S.; Sedelmeier, J.; Van der Eycken, E. V.; Ley, S. V. Angew. Chem. Int. Ed. 2017, 56, 15136-15140.

7. (a) Schwarz, J. L.; Schäfers, F.; Tlahuext-Aca, A.; Lückemeier, L.; Glorius, F. J. Am. Chem. Soc. 2018, 140, 12705-12709; (b) Capaldo, L.; Riccardi, R.; Ravelli, D.; Fagnoni, M. ACS Catal. 2018, 8, 304-309; (c) Vu, M. D.; Das, M.; Liu, X.-W. Chem.: Eur. J. 2017, 23, 1589915902; (d) Matos, J. L. M.; Vásquez-Céspedes, S.; Gu, J.; Oguma, T.; Shenvi, R. A. J. Am. Chem. Soc. 2018, 140, 16976-16981; (e) Pitzer, L.; Sandfort, F.; Strieth-Kalthoff, F.; Glorius, F. J. Am. Chem. Soc. 2017, 139, 13652-13655.

8. Nakajima, M.; Fava, E.; Loescher, S.; Jiang, Z.; Rueping, M. Angew. Chem. Int. Ed. 2015, 54, 8828-8832.

9. Wang, R.; Ma, M.; Gong, X.; Fan, X.; Walsh, P. J. Org. Lett. 2019, 21, 27-31.

10. (a) Fava, E.; Nakajima, M.; Nguyen, A. L. P.; Rueping, M. J. Org. Chem. 2016, 81, 6959-6964; (b) Lee, K. N.; Lei, Z.; Ngai, M.-Y J. Am. Chem. Soc. 2017, 139, 5003-5006; (c) Qi, L.; Chen, Y. Angew. Chem. 2016, 128, 13506-13509.

11. Li, W.; Duan, Y.; Zhang, M.; Cheng, J.; Zhu, C. Chem. Comm. 2016, 52, 7596-7599.

12. Berger, A. L.; Donabauer, K.; König, B. Chem. Sci. 2018, 9 , 7230-7235.

13. Field, J. B.; Ohta, M.; Boyle, C.; Remer, A. N. Engl. J. Med. 1967, 277, 889-894.

14. Lima, F.; Grunenberg, L.; Rahman, H. B. A.; Labes, R.; Sedelmeier, J.; Ley, S. V. Chem. Comm. 2018, 54, 5606-5609.

15. Cambié, D.; Bottecchia, C.; Straathof, N. J. W.; Hessel, V.; Noël, T. Chem. Rev. 2016, 116, 10276-10341.

16. Roibu, A.; Fransen, S.; Leblebici, M. E.; Meir, G.; Van Gerven, T.; Kuhn, S. Sci. Rep. 2018, 8, 5421.

17. (a) Loubière, K.; Oelgemöller, M.; Aillet, T.; Dechy-Cabaret, O.; Prat, L. Chem. Eng. Process. 2016, 104, 120-132; (b) Su, Y.; Straathof, N. J. W.; Hessel, V.; Noël, T. Chem.: Eur. J. 2014, 20, 10562-10589.

18. Xia, Q.; Dong, J.; Song, H.; Wang, Q. Chem.: Eur. J. 2019, 25, 2949-2961.

19. Wagner, P. J.; Truman, R. J.; Puchalski, A. E.; Wake, R. J. Am. Chem. Soc. 1986, 108, 7727-7738.

20. Lowry, M. S.; Goldsmith, J. I.; Slinker, J. D.; Rohl, R.; Pascal, R. A.; Malliaras, G. G.; Bernhard, S. Chem. Mat. 2005, 17, 5712-5719.

21. Wang, S.; Lokesh, N.; Hioe, J.; Gschwind, R. M.; König, B. Chem. Sci. 2019, 10, 4580-4587.

22. (a) Dowd, P.; Zhang, W. Chem. Rev. 1993, 93, 2091-2115; (b) Saladrigas, M.; Bosch, C.; Saborit, G. V.; Bonjoch, J.; Bradshaw, B. Angew. Chem. Int. Ed. 2018, 57, 182-186; (c) Saladrigas, M.; Loren, G.; Bonjoch, J.; Bradshaw, B. ACS Catal. 2018, 8, 11699-11703.

23. Wilsey, S.; Dowd, P.; Houk, K. N. J. Org. Chem. 1999, 64, 88018811.

24. Salamone, M.; Bietti, M. Synlett 2014, 25, 1803-1816.

25. Smith, J. R. L.; Masheder, D. J. Chem. Soc., Perkin Trans. 2 1977, 1732-1736.

26. (a) Dimakos, V.; Su, H. Y.; Garrett, G. E.; Taylor, M. S. J. Am. Chem. Soc. 2019, 141, 5149-5153; (b) Ye, J.; Kalvet, I.; Schoenebeck, F.; Rovis, T. Nature Chemistry 2018, 10, 1037-1041. 
